\title{
A REMARK ON RATIONAL CHEREDNIK ALGEBRAS AND DIFFERENTIAL OPERATORS ON THE CYCLIC QUIVER
}

\author{
IAIN GORDON \\ Department of Mathematics, Glasgow University, Glasgow G12 8QW, Scotland \\ e-mail:ig@maths.gla.ac.uk
}

(Received 30 July, 2005; accepted 10 October, 2005)

\begin{abstract}
We show that the spherical subalgebra $U_{k, c}$ of the rational Cherednik algebra associated to $S_{n}>C_{\ell}$, the wreath product of the symmetric group and the cyclic group of order $\ell$, is isomorphic to a quotient of the ring of invariant differential operators on a space of representations of the cyclic quiver of size $\ell$. This confirms a version of [5, Conjecture 11.22] in the case of cyclic groups. The proof is a straightforward application of work of Oblomkov [12] on the deformed HarishChandra homomorphism, and of Crawley-Boevey, [3] and [4], and Gan and Ginzburg [7] on preprojective algebras.
\end{abstract}

2000 Mathematics Subject Classification. MSC 16U20, 16S32, 13N10.

\section{Introduction.}

1.1. The representation theory of symplectic reflection algebras has links with a number of subjects including algebraic combinatorics, resolutions of singularities, Lie theory and integrable systems. There is a family of symplectic reflection algebras associated to any symplectic vector space $V$ and finite subgroup $\Gamma \leq S p(V)$, but a simple reduction allows one to study those subgroups $\Gamma$ which are generated by symplectic reflections (i.e. by elements whose set of fixed points is of codimension two in $V$ ). This essentially focuses attention on two cases:

(1) $\Gamma=W$, a finite complex reflection group, acting on $V=\mathfrak{h} \oplus \mathfrak{h}^{*}$ where $\mathfrak{h}$ is a reflection representation of $W$

(2) $\Gamma=S_{n}$ 2 $K$, where $K$ is a finite subgroup of $S L_{2}(\mathbb{C})$, acting naturally on $\left(\mathbb{C}^{2}\right)^{n}$.

The representation theory in the first case is mysterious at the moment: several important results are known but there is no general theory yet. On the other hand a geometric point of view on the representation theory in the second case is beginning to emerge. A key fact is that in this case the singular space $V / \Gamma$ admits a crepant resolution of singularities: the representation theory of the symplectic reflection algebra is then expected to be closely related to the resolution. In the case $\Gamma=S_{n}$ (i.e. $K$ is trivial) there are two approaches to this: the first is via noncommutative algebraic geometry [8] the second via sheaves of differential operators [7]. In this paper we extend the second approach to the groups $\Gamma=\Gamma_{n}=S_{n} 2 C_{\ell}$.

The author was partially supported by the Leverhulme trust. 
1.2. To state our result we need to introduce a little notation here. Let $Q$ be the cyclic quiver with $\ell$ vertices and cyclic orientation. Choose an extending vertex (in this case any vertex) 0 . Then let $Q_{\infty}$ be the quiver obtained by adding one vertex named $\infty$ to $Q$ that is joined to 0 by a single arrow.

We shall consider representation spaces of these quivers. Let $\delta=(1,1, \ldots, 1)$ be the affine dimension vector of $Q$, and set $\epsilon=e_{\infty}+n \delta$, a dimension vector for $Q_{\infty}$. Let $\operatorname{Rep}(Q, n \delta)$ and $\operatorname{Rep}\left(Q_{\infty}, \epsilon\right)$ be the representation spaces of these quivers with the given dimension vectors. There is an action of $G=\prod_{r=0}^{\ell-1} G L_{n}(\mathbb{C})$ on both these spaces. In fact, the action of the scalar matrices in $G$ is trivial on $\operatorname{Rep}(Q, n \delta$ ) (but not on $\left.\operatorname{Rep}\left(Q_{\infty}, \epsilon\right)\right)$ and so in this case the action descends to an action of $P G=G / \mathbb{C}^{*}$.

Let $\mathfrak{X}=\operatorname{Rep}(Q, n \delta) \times \mathbb{P}^{n-1}$. There is an action of $P G$ on $\mathfrak{X}$.

1.3. Let $D\left(\operatorname{Rep}\left(Q_{\infty}, \epsilon\right)\right)$ denote the ring of differential operators on the affine space $\operatorname{Rep}\left(Q_{\infty}, \epsilon\right), D_{\mathfrak{X}}(n k)$ the sheaf of twisted differential operators on $\mathfrak{X}$ and $D(\mathfrak{X}, n k)$ its algebra of global sections. The group action of $G$ (respectively $P G)$ on $\operatorname{Rep}\left(Q_{\infty}, \epsilon\right)$ (respectively $\mathfrak{X}$ ) differentiates to an action of $\mathfrak{g}=\operatorname{Lie}(G)$ (respectively $\mathfrak{p g}=\operatorname{Lie}(P G)$ ) by differential operators. This gives mappings

$$
\hat{\tau}: \mathfrak{g} \longrightarrow D\left(\operatorname{Rep}\left(Q_{\infty}, \epsilon\right)\right), \quad \tau: \mathfrak{p g} \longrightarrow D_{\mathfrak{X}}(n k) .
$$

1.4. Let $U_{k, c}$ be the spherical subalgebra of type $S_{n}$. $C_{\ell}$. (This is defined in Section 3.4.)

THEOREM. For all $(k, c)$ there are isomorphisms of algebras

$$
\left(\frac{D\left(\operatorname{Rep}\left(Q_{\infty}, \epsilon\right)\right)}{I_{k, c}}\right)^{G} \cong\left(\frac{D(\mathfrak{X}, n k)}{I_{c}}\right)^{P G} \cong U_{k, c},
$$

where $I_{k, c}$ is the left ideal of $D\left(\operatorname{Rep}\left(Q_{\infty}, \epsilon\right)\right.$ generated by $\left(\hat{\tau}-\chi_{k, c}\right)(\mathfrak{g})$ and $I_{c}$ is the left ideal of $D(\mathfrak{X}, n k)$ generated by $\left(\tau-\chi_{c}\right)(\mathfrak{p g})$ for suitable characters $\chi_{k, c} \in \mathfrak{g}^{*}$ and $\chi_{c} \in \mathfrak{p g}^{*}$. (These are defined in Section 4.)

Note that it is a standard fact that the left hand side is an algebra. The proof of the theorem has two parts. One part constructs a filtered homomorphism from the left hand side to the right hand side using as its main input the work of Oblomkov [12]. The other part proves that the associated graded homomorphism is an isomorphism and is a simple application of results of Crawley-Boevey [3] and [4], and of Gan and Ginzburg [7].

1.5. We give an application of this result in Section 4. For related pairs $(k, c)$ and $\left(k^{\prime}, c^{\prime}\right)$ we construct "shift functors"

$$
U_{k, c^{-}}-\bmod \longrightarrow U_{k^{\prime}, c^{\prime}}-\bmod
$$

using differential operators. We expect these to be a useful tool in the representation theory of Cherednik algebras, deserving of careful study.

1.6. While writing this down, we were informed that the general version of $[\mathbf{5}$, Conjecture 11.22] has been proved in [6]. This result is more general than the work 
presented here and requires a new approach and ideas to overcome problems that simply do not arise for the case $\Gamma=S_{n} 2 C_{\ell}$.

\section{Quivers.}

2.1. Once and for all fix integers $\ell$ and $n$. We assume that both are greater than 1. Set $\eta=\exp (2 \pi i / \ell)$.

2.2. Let $Q$ be the cyclic quiver with $\ell$ vertices and cyclic orientation. Choose an extending vertex (in this case any vertex) 0 . Then let $Q_{\infty}$ be the quiver obtained by adding one vertex named $\infty$ to $Q$ that is joined to 0 by a single arrow. Let $\bar{Q}$ and $\bar{Q}_{\infty}$ denote the double quivers of $Q$ and $Q_{\infty}$ respectively, obtained by inserting an arrow $a^{*}$ in the opposite direction to every arrow $a$ in the quiver.

We shall consider representation spaces of these quivers. Let $\delta=(1,1, \ldots, 1)$ be the affine dimension vector of $Q$, and set $\epsilon=e_{\infty}+n \delta$, a dimension vector for $Q_{\infty}$. Recall that

$$
\operatorname{Rep}(Q, n \delta)=\bigoplus_{r=0}^{\ell-1} \operatorname{Mat}_{n}(\mathbb{C})=\left\{\left(X_{0}, X_{1}, \ldots, X_{\ell-1}\right): X_{r} \in \operatorname{Mat}_{n}(\mathbb{C})\right\}=\{(X)\}
$$

and

$$
\begin{aligned}
\operatorname{Rep}\left(Q_{\infty}, \epsilon\right) & =\bigoplus_{r=0}^{\ell-1} \operatorname{Mat}_{n}(\mathbb{C}) \oplus \mathbb{C}^{n}=\left\{\left(X_{0}, X_{1}, \ldots, X_{\ell-1}, i\right): X_{r} \in \operatorname{Mat}_{n}(\mathbb{C}), i \in \mathbb{C}^{n}\right\} \\
& =\{(X, i)\} .
\end{aligned}
$$

Let $G=\prod_{r=0}^{\ell-1} G L_{n}(\mathbb{C})$ be the base change group. If $g=\left(g_{0}, \ldots, g_{\ell-1}\right)$, then $g$ acts on $\operatorname{Rep}(Q, n \delta)$ by

$$
g \cdot\left(X_{0}, X_{1}, \ldots, X_{\ell-1}\right)=\left(g_{0} X_{0} g_{1}^{-1}, g_{1} X_{1} g_{2}^{-1}, \ldots, g_{\ell-1} X_{\ell-1} g_{0}^{-1}\right)
$$

and on $\operatorname{Rep}\left(Q_{\infty}, \epsilon\right)$ by

$$
g \cdot\left(X_{0}, X_{1}, \ldots, X_{\ell-1}, i\right)=\left(g_{0} X_{0} g_{1}^{-1}, g_{1} X_{1} g_{2}^{-1}, \ldots, g_{\ell-1} X_{\ell-1} g_{0}^{-1}, g_{0} i\right) .
$$

The action of the scalar subgroup $\mathbb{C}^{*}$ is trivial in the first action (but not the second) and so we can consider the first action, as a $P G$-action where $P G=G / \mathbb{C}^{*}$. Let $\mathfrak{g}$ and $\mathfrak{p g}$ be the Lie algebras of $G$ and $P G$, respectively.

2.3. Let $\mathfrak{h}^{\text {reg }} \subset \mathbb{C}^{n}$ be the affine open subvariety consisting of points $x=$ $\left(x_{1}, \ldots, x_{n}\right)$ such that

(i) if $i \neq j$ then $x_{i} \neq \eta^{m} x_{j}$ for all $m \in \mathbb{Z}$,

(ii) for each $1 \leq i \leq n x_{i} \neq 0$.

This is the subset of $\mathbb{C}^{n}$ on which $\Gamma_{n}=S_{n}$ 2 $C_{\ell}$ acts freely. 
2.4. We can embed $\mathfrak{h}^{\text {reg }}$ into $\operatorname{Rep}(Q, n \delta)$ by first considering a point $x=$ $\left(x_{1}, \ldots, x_{n}\right) \in \mathfrak{h}^{\text {reg }}$ as a diagonal matrix $X=\operatorname{diag}\left(x_{1}, \ldots, x_{n}\right)$ and then sending this to $\underline{X}=(X, X, \ldots, X)$. We denote the image of $\mathfrak{h}^{\text {reg }}$ in $\operatorname{Rep}(Q, n \delta)$ by $\mathcal{S}$.

Let $T_{\Delta}$ be the subgroup of $G$ with elements $(T, T, \ldots, T)$ where $T$ is a diagonal matrix in $G L_{n}(\mathbb{C})$. Then $T_{\Delta}$ is the stabiliser of $\mathcal{S}$. Now consider the mapping

$$
\pi: G / T_{\Delta} \times \mathfrak{h}^{\text {reg }} \longrightarrow \operatorname{Rep}(Q, n \delta)
$$

given by $\pi\left(g T_{\Delta}, x\right)=g \cdot \underline{X}$. If we let $G$ act on $G / T_{\Delta} \times \mathfrak{h}^{\text {reg }}$ by left multiplication, then $\pi$ is a $G$-equivariant mapping.

LEMMA. $\pi$ is an étale mapping with covering group $\Gamma_{n}$. In fact, its image $\operatorname{Rep}(Q, n \delta)^{\text {reg }}$ is open in $\operatorname{Rep}(Q, n \delta)$ and we have an isomorphism

$$
\omega: G / T_{\Delta} \times_{\Gamma_{n}} \mathfrak{h}^{\text {reg }} \longrightarrow \operatorname{Rep}(Q, n \delta)^{\text {reg }} .
$$

Proof. Let $\mathcal{S}=\left\{\underline{X}: x \in \mathfrak{h}^{\text {reg }}\right\}$. Set $N_{G}(\mathcal{S})=\{g \in G: g \cdot \mathcal{S}=\mathcal{S}\}$ and

$$
Z_{G}(\mathcal{S})=\{g \in G: g \cdot \underline{X}=\underline{X} \text { for all } \underline{X} \in \mathcal{S}\} .
$$

Suppose that $g \cdot \underline{X}=\underline{Y}$ for some $\underline{X}, \underline{Y} \in \mathcal{S}$. This implies that for each $0 \leq i \leq \ell-1$

$$
g_{i} \operatorname{diag}(x)^{\ell} g_{i}^{-1}=\operatorname{diag}(y)^{\ell} .
$$

The hypotheses on $\mathfrak{h}^{\text {reg }}$ imply that both $\operatorname{diag}(x)^{\ell}$ and $\operatorname{diag}(y)^{\ell}$ are regular semisimple in $\mathrm{Mat}_{n}(\mathbb{C})$. Two such elements are conjugate if and only if $g_{i} \in N_{G L_{n}(\mathbb{C})}(T)=T \cdot S_{n}$, where $T$ is the diagonal subgroup of $G L_{n}(\mathbb{C})$. Hence there exists $\sigma \in S_{n}$ such that for all $i$ we have $g_{i}=t_{i} \sigma$ for some $t_{i} \in T$, and for all $1 \leq r \leq n$ we have that $x_{\sigma(r)}^{\ell}=y_{r}^{\ell}$. Hence $x_{\sigma(r)}=\eta^{m_{r}} y_{r}$ for some $m_{r} \in \mathbb{Z}$. Now we find that $\underline{Y}=g \cdot \underline{X}$ implies that $\operatorname{diag}\left(y_{r}\right)=$ $t_{i} t_{i+1}^{-1} \operatorname{diag}\left(\eta^{m_{r}} y_{r}\right)$. Since $y_{r} \neq 0$ this shows that $t_{i+1}=\operatorname{diag}\left(\eta^{m_{r}}\right) t_{i}$ for each $i$. Hence we find that $g T_{\Delta}=\left(\sigma, \operatorname{diag}\left(\eta^{m_{r}}\right) \sigma, \ldots, \operatorname{diag}\left(\eta^{m_{r}}\right)^{\ell-1} \sigma\right) T_{\Delta}$.

In particular, if $\underline{X}=\underline{Y}$ we see from above that each $m_{r}=0$, so that $Z_{G}(\mathcal{S})=T_{\Delta}$. Thus the group $\Gamma_{n}$ is isomorphic to $N_{G}(\mathcal{S}) / Z_{G}(\mathcal{S})$ via the homomorphism that sends $\left(\eta^{m_{1}}, \ldots, \eta^{m_{r}}\right) \sigma$ to $\left(\sigma, \operatorname{diag}\left(\eta^{m_{r}}\right) \sigma, \ldots, \operatorname{diag}\left(\eta^{m_{r}}\right)^{\ell-1} \sigma\right) T_{\Delta}$.

Now suppose that $\pi\left(g T_{\Delta}, x\right)=\pi\left(h T_{\Delta}, y\right)$. Then $\left(h^{-1} g\right) \cdot \underline{X}=\underline{Y}$ and so we see that $h^{-1} g \in N_{G}(\mathcal{S})$. This shows that $\pi$ is the composition

$$
G / T_{\Delta} \times \mathfrak{h}^{\text {reg }} \longrightarrow G / T_{\Delta} \times_{\Gamma_{n}} \mathfrak{h}^{\text {reg }} \stackrel{\sim}{\longrightarrow} \operatorname{Rep}(Q, n \delta)^{r e g} .
$$

The first mapping factors out the action of $\Gamma_{n}$, and since $\Gamma_{n}$ acts freely on $\mathfrak{h}^{\text {reg }}$ this is an étale mapping. Hence, to prove the lemma, it suffices to show that $\operatorname{Rep}(Q, n \delta)^{\text {reg }}$ is open in $\operatorname{Rep}(Q, n \delta)$.

We claim first that $\operatorname{Rep}(Q, n \delta)^{r e g}$ is the set $O$ of representations of $Q$ that decompose into $n$ simple modules of dimension $\delta$ and whose endomorphism ring is $n$-dimensional. To prove this, observe that any element of $\operatorname{Rep}(Q, n \delta)^{r e g}$ is isomorphic to a representation of the form $\underline{X}$ and so it decomposes into the $n$ indecomposable modules $\underline{X}_{1}, \ldots \underline{X}_{n}$ of dimension $\delta$, where $\underline{X}_{i}=\left(x_{i}, x_{i}, \ldots, x_{i}\right)$. (The condition $x_{i} \neq 0$ implies simplicity.) Now the representation $\underline{X}_{i}$ is isomorphic to the representation $\left(1,1, \ldots, 1, x_{i}^{\ell}\right)$. By hypothesis $x_{i}^{\ell} \neq x_{j}^{\ell}$ and so we deduce that the representations $\underline{x}_{i}$ are pairwise non-isomorphic which ensures that the endomorphism ring of $X$ is $n$ dimensional. This proves the inclusion $\operatorname{Rep}(Q, n \delta)^{r e g} \subseteq O$. On the other hand, if $V$ belongs to $O$ then $V=V_{1} \oplus \ldots \oplus V_{n}$, where each $V_{i}$ is isomorphic to a representation 
$\left(1,1, \ldots, 1, v_{i}\right)$, for some non-zero scalars $v_{i}$. Moreover, $\operatorname{since} \operatorname{dim} \operatorname{End}(V)=n$ the $v_{i}$ must be pairwise distinct. Now, let $\eta_{i}$ be an $\ell$-th root of $v_{i}$. Then $V_{i}$ is isomorphic to $\left(\eta_{i}, \ldots, \eta_{i}\right)$. Therefore $V$ is isomorphic to the representation $\underline{X}$, where $x=\left(\eta_{1}, \ldots, \eta_{n}\right)$.

Now we must show that $O$ is open $\operatorname{in} \operatorname{Rep}(Q, n \delta)$. We use first the fact that the canonical decomposition of the vector $n \delta$ is $\delta+\delta+\cdots+\delta$, [13, Theorem 3.6]. This means that the representations of $\operatorname{Rep}(Q, n \delta)$ whose indecomposable components all have dimension $\delta$ form an open set. Now, consider the morphism $f$ from $\operatorname{Rep}(Q, \delta)$ to $\mathbb{C}$ that sends the representation $\left(\lambda_{1}, \ldots, \lambda_{\ell}\right)$ to the product $\lambda_{1} \ldots \lambda_{\ell}$. The open set $f^{-1}\left(\mathbb{C}^{*}\right)$ consists of the simple representations of dimension vector $\delta$. Therefore the subset of $\operatorname{Rep}(Q, n \delta)$ consisting of representations which decompose as the sum of $n$ simple representations of dimension vector $\delta$ is open. On the other hand, the function from $\operatorname{Rep}(Q, n \delta)$ to $\mathbb{N}$ that sends a representation $V$ to $\operatorname{dim} \operatorname{End}(V)$ is upper semicontinuous. Thus $\{V: \operatorname{dim} \operatorname{End}(v) \leq n\}$ is an open set in $\operatorname{Rep}(Q, n \delta)$. Intersecting these two sets shows that $O$ is open, as required.

2.5. Now we are going to move from $Q$ to $Q_{\infty}$ and so we start with the following inclusion:

$$
\left\{\left(\left[g T_{\Delta}, x\right], i\right): g_{0}^{-1} i \text { is a cyclic vector for } \operatorname{diag}(x)\right\} \subset\left(G / T_{\Delta} \times_{\Gamma_{n}} \mathfrak{h}^{\text {reg }}\right) \times \mathbb{C}^{n}
$$

By applying $\omega^{-1} \times \mathrm{id}_{\mathbb{C}^{n}}$, the left-hand side corresponds to an open subset of $\operatorname{Rep}(Q, n \delta) \times \mathbb{C}^{n}=\operatorname{Rep}\left(Q_{\infty}, \epsilon\right)$. Call that set $U_{\infty}$. This is a $G$-invariant open set since the $G$-action on triples is given by

$$
h \cdot\left(\left[g T_{\Delta}, x\right], i\right)=\left(\left[h g T_{\Delta}, x\right], h_{0} i\right)
$$

so that $g_{0}^{-1} i$ is cyclic for $\operatorname{diag}(x)$ if and only if $\left(h_{0} g_{0}\right)^{-1} h_{0} i$ is cyclic for $\operatorname{diag}(x)$. Observe too that $U_{\infty}$ is an affine variety. Indeed it is defined by the non-vanishing of the morphism

$$
s:\left(G / T_{\Delta} \times_{\Gamma_{n}} \mathfrak{h}^{\text {reg }}\right) \times \mathbb{C}^{n} \longrightarrow \mathbb{C}
$$

which sends $\left(\left[g T_{\Delta}, x\right], i\right)$ to $\left(g_{0}^{-1} i\right) \wedge \operatorname{diag}(x) \cdot\left(g_{0}^{-1} i\right) \wedge \cdots \wedge \operatorname{diag}(x)^{n-1} \cdot\left(g_{0}^{-1} i\right)$.

LEMMA. The G-action on $U_{\infty}$ is free and projection onto the second component

$$
\pi_{2}: U_{\infty} \longrightarrow \mathfrak{h}^{r e g} / \Gamma_{n}
$$

is a principal G-bundle.

Proof. Suppose that $h \cdot\left(\left[g T_{\Delta}, x\right], i\right)=\left(\left[g T_{\Delta}, x\right], i\right)$. Then $\left[g^{-1} h g T_{\Delta}, x\right]=\left[T_{\Delta}, x\right]$ and so, by Lemma $2.4, g^{-1} h g \in T_{\Delta}$.

We have that $h_{0} i=i$. Setting $i^{\prime}=g_{0}^{-1} i$ implies that $g_{0}^{-1} h_{0} g_{0} i^{\prime}=i^{\prime}$. By hypothesis $i^{\prime}$ is a cyclic vector for $\operatorname{diag}(x)$. Hence with respect to the standard basis $\left\{e_{j}\right\}, i^{\prime}$ decomposes as $\sum \lambda_{j} e_{j}$, where each $\lambda_{j}$ is non-zero. Therefore the only diagonal matrix that fixes $i^{\prime}$ is the identity element. In other words $g_{0}^{-1} h_{0} g_{0}=I_{n}$. Since $g^{-1} h g \in T_{\Delta}$ this implies that $g^{-1} h g=\mathrm{id}$. Thus $h=\mathrm{id}$ and this proves that the action is free.

It remains to prove that each fibre of $\pi_{2}$ is a $G$-orbit. We take $\left(\left[g T_{\Delta}, x\right], i\right) \in$ $\pi_{2}^{-1}([x])$. This equals $g \cdot\left(\left[T_{\Delta}, x\right], g_{0}^{-1} i\right)$. Now $g_{0}^{-1} i$ is a cyclic vector for $\operatorname{diag}(x)$ and so it has the form $\sum \lambda_{j} e_{j}$ with each $\lambda_{j}$ non-zero. Let $t=\operatorname{diag}\left(\lambda_{1}, \ldots, \lambda_{n}\right)$ and consider 
$\underline{t}=(t, \ldots, t) \in T_{\Delta}$. We have

$$
\left(\left[g T_{\Delta}, x\right], i\right)=g \underline{t t}^{-1}\left(\left[T_{\Delta}, x\right], g_{0}^{-1} i\right)=g \underline{t}\left(\left[T_{\Delta}, x\right], \sum_{j=1}^{n} e_{j}\right) .
$$

This proves that each fibre of $\pi_{2}$ is indeed a $G$-orbit.

2.6. Consider the representation space for the doubled quiver $\bar{Q}_{\infty}$ :

$$
\begin{aligned}
\operatorname{Rep}\left(\bar{Q}_{\infty}, \epsilon\right) & =\left\{\left(X_{0}, \ldots, X_{\ell-1}, Y_{0}, \ldots, Y_{\ell-1}, i, j\right): X_{r}, Y_{r} \in \operatorname{Mat}_{n}(\mathbb{C}), i \in \mathbb{C}^{n}, j \in\left(\mathbb{C}^{*}\right)^{n}\right\} \\
& =\{(X, Y, i, j)\}
\end{aligned}
$$

We can naturally identify it with $T^{*} \operatorname{Rep}\left(Q_{\infty}, \epsilon\right)$. The group $G$ acts on the base and hence on the total space of the cotangent bundle. The resulting moment map

$$
\mu: \operatorname{Rep}\left(\bar{Q}_{\infty}, \epsilon\right) \longrightarrow \mathfrak{g}^{*} \cong \mathfrak{g}
$$

is given by

$$
\mu(X, Y, i, j)=[X, Y]+i j .
$$

Theorem (Gan-Ginzburg, Crawley-Boevey). Let $\mu^{-1}(0)$ denote the schemetheoretic fibre of $\mu$.

(1) $\mu^{-1}(0)$ is reduced, equidimensional and a complete intersection.

(2) The moment map $\mu$ is flat.

(3) $\mathbb{C}\left[\mu^{-1}(0)\right]^{G} \cong \mathbb{C}\left[\mathfrak{h} \oplus \mathfrak{h}^{*}\right]^{\Gamma_{n}}$.

Proof. (i) This is proved in [7, Theorem 3.2.3].

(ii) This follows from [3, Theorem 1.1] and the dimension formula in [7, Theorem 3.2.3(iii)].

(iii) This is $[\mathbf{4}$, Theorem 1.1].

2.7. Let $\mathfrak{X}=\left\{(X, i) \in \operatorname{Rep}(Q, n \delta) \times \mathbb{P}^{n-1}\right\}$. This space is the quotient of the (quasi-affine) open subvariety

$$
U=\{(X, i): i \neq 0\} \subset \operatorname{Rep}\left(Q_{\infty}, \epsilon\right)
$$

by the scalar group $\mathbb{C}^{*}$. Thus there is an action of $P G$ on $\mathfrak{X}$.

Since

$$
T^{*} \mathbb{P}^{n-1}=\{(i, j): i \neq 0, j i=0\} / \mathbb{C}^{*}
$$

we have

$$
T^{*} \mathfrak{X}=\left\{(X, Y, i, j) \in \operatorname{Rep}\left(\bar{Q}_{\infty}, \epsilon\right): i \neq 0, j i=0\right\} / \mathbb{C}^{*} .
$$

The $P G$ action on $\mathfrak{X}$ gives rise to a moment map

$$
\mu_{\mathfrak{X}}: T^{*} \mathfrak{X} \longrightarrow \mathfrak{p g}^{*} \cong \mathfrak{p g} .
$$


Let

$$
\mu_{\mathfrak{X}}^{-1}(0)=\left\{(X, Y, i, j) \in \operatorname{Rep}\left(\bar{Q}_{\infty}, \epsilon\right): i \neq 0, j i=0, \quad[X, Y]+i j=0\right\} / \mathbb{C}^{*}
$$

denote the scheme theoretic fibre of 0 .

Proposition. There is an isomorphism $\mathbb{C}\left[\mu_{\mathfrak{X}}^{-1}(0)\right]^{P G} \cong \mathbb{C}\left[\mathfrak{h} \oplus \mathfrak{h}^{*}\right]^{\Gamma_{n}}$.

Proof. Consider the $G$-equivariant open subvariety of $\mu^{-1}(0)$ given by the nonvanishing of $i$. The variety $\mu^{-1}(0)$ is determined by the conditions $[X, Y]+i j=0$, and so if we take the trace of this equation then we see that $0=\operatorname{Tr}(i j)=\operatorname{Tr}(j i)=j i$. Thus $\left\{(X, Y, i, j) \in \operatorname{Rep}\left(\bar{Q}_{\infty}, \epsilon\right): i \neq 0, j i=0\right\} \cap \mu^{-1}(0)$ is an open subvariety of $\mu^{-1}(0)$ and so, in particular, is reduced by Theorem 2.6(1). Hence factoring out by the action of $\mathbb{C}^{*} \leq G$ shows that $\mu_{\mathfrak{X}}^{-1}(0)$ is reduced and that there is a $P G$-equivariant morphism

$$
\mu_{\mathfrak{X}}^{-1}(0) \longrightarrow \mu^{-1}(0) / / \mathbb{C}^{*}
$$

This induces an algebra map

$$
\alpha: \mathbb{C}\left[\mu^{-1}(0)\right]^{G} \longrightarrow \mathbb{C}\left[\mu_{\mathfrak{X}}^{-1}(0)\right]^{P G} .
$$

We now follow some of the proof of [7, Lemma 6.3.2]. Write $\mathrm{O}_{1}$ for the conjugacy class of rank one nilpotent matrices in $\mathfrak{g l}(n)$, and let $\overline{\mathrm{O}}_{1}$ denote the closure of $\mathrm{O}_{1}$ in $\mathfrak{g l}(n)$. The moment map $v: T^{*} \mathbb{P}^{n-1} \longrightarrow \mathfrak{g l}(n)^{*} \cong \mathfrak{g l}(n)$ that sends $(i, j)$ to $i j$ gives a birational isomorphism $T^{*} \mathbb{P}^{n-1} \longrightarrow \overline{\mathrm{O}}_{1}$. Let $J \subset \mathbb{C}[\mathfrak{g l}(n)]=C[Z]$ be the ideal generated by all $2 \times 2$ minors of the matrix $Z$ and also by the trace function. Then $J$ is a prime ideal whose zero scheme is $\overline{\mathrm{O}}_{1}$ and the pullback morphism $v^{*}: \mathbb{C}[\mathfrak{g l}(n)] / J \longrightarrow \mathbb{C}\left[T^{*} \mathbb{P}^{n-1}\right]$ is a graded isomorphism.

Now the moment map $\mu_{\mathfrak{X}}: T^{*} \mathfrak{X} \longrightarrow \mathfrak{g}^{*}$ factors as the composite

$$
T^{*} \mathfrak{X}=T^{*} \operatorname{Rep}(Q, n \delta) \times T^{*} \mathbb{P}^{n-1} \longrightarrow T^{*} \operatorname{Rep}(Q, n \delta) \times \overline{\mathrm{O}}_{1} \stackrel{\theta}{\longrightarrow} \mathfrak{p g}^{*},
$$

where the first mapping is id $\times v$ and the second mapping $\theta$ sends $(X, Y, Z)$ to $[X, Y]+$ $Z_{0}$, where $Z_{0}$ indicates that we place the matrix $Z$ on the copy of $\mathfrak{g l}(n)$ associated to the vertex 0 . We have a graded algebra isomorphism

$$
\mathbb{C}\left[T^{*} \operatorname{Rep}(Q, n \delta)\right] \otimes \mathbb{C}[\mathfrak{g l}(n)] / J \longrightarrow \mathbb{C}\left[T^{*} \mathfrak{X}\right] .
$$

Now write $\mathbb{C}[X, Y, Z]=\mathbb{C}\left[T^{*} \operatorname{Rep}(Q, n \delta) \times \mathfrak{g l}(n)\right]$, and let $\mathbb{C}[X, Y, Z]\left([X, Y]+Z_{0}\right)$ denote the ideal in $\mathbb{C}[X, Y, Z]$ generated by all matrix entries of the $\ell$ matrices $[X, Y]+$ $Z_{0}$. Let $\mathbf{I}$ denote the ideal $\mathbb{C}[X, Y, Z]\left([X, Y]+Z_{0}\right)+\mathbb{C}[X, Y] \otimes J \subset \mathbb{C}[X, Y, Z]$. From the above we have

$$
\mathbb{C}\left[\mu_{\mathfrak{X}}^{-1}(0)\right] \cong \mathbb{C}\left[T^{*} \operatorname{Rep}(Q, n \delta) \times \overline{\mathrm{O}}_{1}\right] / \mathbb{C}\left[T^{*} \operatorname{Rep}(Q, n \delta) \times \overline{\mathrm{O}}_{1}\right] \theta^{*}(\mathfrak{g l}(n))=\mathbb{C}[X, Y, Z] / \mathbf{I} .
$$

Define an algebra homomorphism $r: \mathbb{C}[X, Y, Z] \longrightarrow \mathbb{C}[X, Y]$ by sending $P \in$ $\mathbb{C}[X, Y, Z]$ to the function $(X, Y) \mapsto P\left(X, Y,-[X, Y]_{0}\right)$. Obviously $r$ induces an isomorphism $\mathbb{C}[X, Y, Z] / \mathbb{C}[X, Y, Z]\left([X, Y]+Z_{0}\right) \cong \mathbb{C}[X, Y] / I_{1}$, where $I_{1}$ is the ideal of $\mathbb{C}[\operatorname{Rep}(\bar{Q}, n \delta)]=\mathbb{C}[X, Y]$ generated by the elements

$$
\sum_{h(a)=i} X_{a} X_{a^{*}}-\sum_{t(a)=i} X_{a^{*}} X_{a}
$$


for all $i$ not equal to zero. Observe that the linear function $P:(X, Y, Z) \mapsto \operatorname{Tr} Z=$ $\operatorname{Tr}\left([X, Y]+Z_{0}\right)$ belongs to the ideal $\mathbb{C}[X, Y, Z]\left([X, Y]+Z_{0}\right)$. We deduce that the mapping $r$ sends $\mathbb{C}[X, Y] \otimes J$ to the ideal generated by

$$
\operatorname{rank}\left(\sum_{h(a)=0} X_{a} X_{a^{*}}-\sum_{t(a)=0} X_{a^{*}} X_{a}\right) \leq 1 .
$$

Thus we obtain algebra isomorphisms

$$
\mathbb{C}\left[\mu_{\mathfrak{X}}^{-1}(0)\right] \cong \mathbb{C}[X, Y, Z] / \mathbf{I} \cong \mathbb{C}\left[T^{*} \operatorname{Rep}(Q, n \delta)\right] / I_{2},
$$

where $I_{2}$ is the ideal generated by the elements

$$
\sum_{h(a)=i} X_{a} X_{a^{*}}-\sum_{t(a)=i} X_{a^{*}} X_{a}
$$

for all $1 \leq i \leq \ell-1$, and

$$
\operatorname{rank}\left(\sum_{h(a)=0} X_{a} X_{a^{*}}-\sum_{t(a)=0} X_{a^{*}} X_{a}\right) \leq 1
$$

By [10, Theorem 1] the $G$-invariant (respectively $P G$-invariant) elements of $\mathbb{C}\left[\operatorname{Rep}\left(\bar{Q}_{\infty}, \epsilon\right)\right]($ respectively $\mathbb{C}[\operatorname{Rep}(\bar{Q}, n \delta)])$ are generated by traces along oriented cycles. Since all oriented cycles in $\bar{Q}$ are oriented cycles in $\bar{Q}_{\infty}$ we have a surjective composition of algebra homomorphisms

$$
\mathbb{C}\left[\mathfrak{h} \oplus \mathfrak{h}^{*}\right]^{\Gamma_{n}} \cong \mathbb{C}\left[\mu^{-1}(0)\right]^{G} \longrightarrow \mathbb{C}\left[\mu_{\mathfrak{X}}^{-1}(0)\right]^{P G} \longrightarrow\left(\frac{\mathbb{C}[\operatorname{Rep}(\bar{Q}, n \delta)]}{I_{2}}\right)^{P G},
$$

where the first isomorphism is Theorem 2.6(3). The left hand side is a domain of dimension $2 \operatorname{dim} \mathfrak{h}$ and so, to see that the mapping is an isomorphism, it suffices to prove that the right hand side also has dimension $2 \operatorname{dim} \mathfrak{h}$.

Let $I_{3}$ be the ideal of $\mathbb{C}[\operatorname{Rep}(\bar{Q}, n \delta)]$ generated by the elements

$$
\sum_{h(a)=i} X_{a} X_{a^{*}}-\sum_{t(a)=i} X_{a^{*}} X_{a}
$$

for all $i$. This is the ideal of the zero fibre of the moment map for the $P G$-action on $\operatorname{Rep}(\bar{Q}, n \delta)$. This ideal contains $I_{2}$ since the rank condition on the matrices is implied by the commutator condition. Hence there is a surjective mapping

$$
\frac{\mathbb{C}[\operatorname{Rep}(\bar{Q}, n \delta)]^{P G}}{I_{2}^{P G}} \longrightarrow \frac{\mathbb{C}[\operatorname{Rep}(\bar{Q}, n \delta)]^{P G}}{I_{3}^{P G}} .
$$

We do not know yet whether the right hand side is reduced or not, but by [4, Theorem 1.1] the reduced quotient of the right hand side is the ring of functions of the variety $\left(\mathfrak{h} \oplus \mathfrak{h}^{*}\right) / \Gamma_{n}$. As this variety has dimension $2 \operatorname{dim} \mathfrak{h}$ we deduce that the composition in (2.7.1) is an isomorphism, and hence that

$$
\mathbb{C}\left[\mu_{\mathfrak{X}}^{-1}(0)\right]^{P G} \cong \mathbb{C}\left[\mathfrak{h} \oplus \mathfrak{h}^{*}\right]^{\Gamma_{n}} .
$$


REMARK. In passing let us note that the commutativity of the following diagram

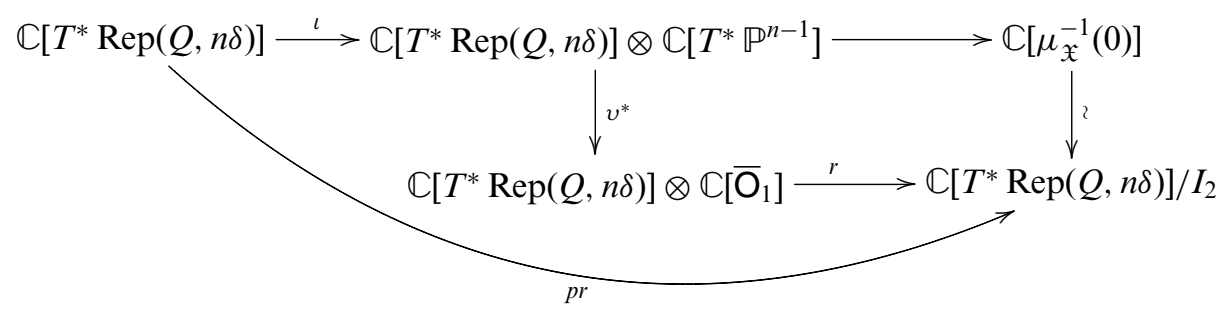

where $\iota(f)=f \otimes 1$, shows that im $\iota$ maps surjectively onto $\mathbb{C}\left[\mu_{\mathfrak{X}}^{-1}(0)\right]$.

\section{Differential operators.}

3.1. Symplectic reflection algebras. Let $C_{\ell}$ be the cyclic subgroup of $S L_{2}(\mathbb{C})$ generated by $\sigma=\operatorname{diag}\left(\eta, \eta^{-1}\right)$. The vector space $V=\left(\mathbb{C}^{2}\right)^{n}$ admits an action of $S_{n}$ 々 $C_{\ell}=S_{n} \ltimes\left(C_{\ell}\right)^{n}$. Here $\left(C_{\ell}\right)^{n}$ acts by extending the natural action of $C_{\ell}$ on $\mathbb{C}^{2}$, whilst $S_{n}$ acts by permuting the $n$ copies of $\mathbb{C}^{2}$. For an element $\gamma \in C_{\ell}$ and an integer $1 \leq i \leq n$ we write $\gamma_{i}$ to indicate the element $(1, \ldots, \gamma, \ldots, 1) \in\left(C_{\ell}\right)^{n}$ which is nontrivial in the $i$-th factor.

3.2. The elements $S_{n}$ 2 $C_{\ell}$ whose fixed points are a subspace of codimension two in $V$ are called symplectic reflections. In this case their conjugacy classes are of two types.

(S) The elements $s_{i j} \gamma_{i} \gamma_{j}^{-1}$ where $1 \leq i, j \leq n, s_{i j} \in S_{n}$ is the transposition that swaps $i$ and $j$, and $\gamma \in C_{\ell}$.

$\left(C_{\ell}\right)$ The elements $\gamma_{i}$ for $1 \leq i \leq n$ and $\gamma \in C_{\ell} \backslash\{1\}$.

There is a unique conjugacy class of type $(S)$ and $\ell-1$ of type $\left(C_{\ell}\right)$ (depending on the non-trivial element we choose from $C_{\ell}$ ). We shall consider a conjugation invariant function from the set of symplectic reflections to $\mathbb{C}$. We can identify it with a pair $(k, c)$ where $k \in \mathbb{C}$ and $c$ is an $(\ell-1)$-tuple of complex numbers: the function sends elements from $(S)$ to $k$ and the elements $\left(\sigma^{m}\right)_{i}$ to $c_{m}$.

3.3. There is a symplectic form on $V$ that is induced from $n$ copies of the standard symplectic form $\omega$ on $\mathbb{C}^{2}$. If we pick a basis $\{x, y\}$ for $\mathbb{C}^{2}$ such that $\omega(x, y)=1$, then we can extend this naturally to a basis $\left\{x_{i}, y_{i}: 1 \leq i \leq n\right\}$ of $V$ such that the $x$ 's and the $y$ 's form Lagrangian subspaces and $\omega\left(x_{i}, y_{j}\right)=\delta_{i j}$. We let $T V$ denote the tensor algebra on $V$ : with our choice of basis this is just the free algebra on generators $x_{i}, y_{i}$ for $1 \leq i \leq n$. The symplectic reflection algebra $H_{k, c}$ associated to $S_{n}$ 乙 $C_{\ell}$ is the quotient of $T V *\left(S_{n} 2 C_{\ell}\right)$ by the following relations:

$$
\begin{array}{rlrl}
x_{i} x_{j} & =x_{j} x_{i}, \quad y_{i} y_{j}=y_{j} y_{i} & & (\text { for all } 1 \leq i, j \leq n), \\
y_{i} x_{i}-x_{i} y_{i}=1+k \sum_{j \neq i} \sum_{\gamma \in C_{\ell}} s_{i j} \gamma_{i} \gamma_{j}^{-1}+\sum_{\gamma \in C_{\ell} \backslash\{1\}} c_{\gamma} \gamma_{i} & (\text { for } 1 \leq i \leq n), \\
y_{i} x_{j}-x_{j} y_{i}=-k \sum_{m=0}^{\ell-1} \eta^{m} s_{i j}\left(\sigma^{m}\right)_{i}\left(\sigma^{m}\right)_{j}^{-1} & & (\text { for } i \neq j) .
\end{array}
$$

(NB: my $k$ is $-k$ for Oblomkov.) 
3.4. The spherical algebra. The symmetrising idempotent of the group algebra $C\left(S_{n} 2 C_{\ell}\right)$ is given by

$$
e=\frac{1}{\left|S_{n} 2 C_{\ell}\right|} \sum_{w \in S_{n} C_{\ell}} w .
$$

The subalgebra $e H_{k, c} e$ is denoted by $U_{k, c}$ and called the spherical algebra. It will be our main object of study.

3.5. Rings of differential operators. Recall the definition of $\mathfrak{X}$ from 2.7 . Let $D_{\mathfrak{X}}(n k)$ denote the sheaf of twisted differential operators on $\mathfrak{X}$ and let $D(\mathfrak{X}, n k)$ be its algebra of global sections. This is simply the tensor product $D(\operatorname{Rep}(Q, n \delta)) \otimes D_{\mathbb{P} n-1}(n k)$. (The twisted differential operators on $\mathbb{P}^{n-1}$ can be defined as follows. Let $A_{n}=$ $\mathbb{C}\left[x_{1}, \ldots, x_{n}, \partial_{1}, \ldots, \partial_{n}\right]$ be the $n$-th Weyl algebra. This is a graded algebra with $\operatorname{deg}\left(x_{i}\right)=1$ and $\operatorname{deg}\left(\partial_{i}\right)=-1$. The degree zero component is the subring generated by the operators $x_{i} \partial_{j}$ which, under the commutator, generate the Lie algebra $\mathfrak{g l}(n)$. Call this subring $R$. Let $\mathbf{E}=\sum_{i=1}^{n} x_{i} \partial_{i} \in R$ be the Euler operator. Then $D\left(\mathbb{P}^{n-1}, n k\right)$ is the quotient of $R$ by the two-sided ideal generated by $\mathbf{E}-n k$.)

The group action of $P G$ on $\mathfrak{X}$ differentiates to an action of $\mathfrak{p g}$ on $\mathfrak{X}$ by differential operators. This gives a mapping

$$
\tau: \mathfrak{p g} \longrightarrow D_{\mathfrak{X}}(n k) .
$$

(One way to understand this is to start back with $U \subset \operatorname{Rep}\left(Q_{\infty}, \epsilon\right)$ and look at the $G$ action on $U$. Differentiating the $G$-action gives an action of $\mathfrak{g}$ by differential operators on $U, \hat{\tau}: \mathfrak{g} \longrightarrow D_{U}$. Since $\mathbb{C}^{*}$ acts trivially on $\operatorname{Rep}(Q, n \delta)$ and by scaling on $i \in \operatorname{Rep}\left(Q_{\infty}, \epsilon\right)$, we find that $\hat{\tau}(\mathrm{id})=1 \otimes \mathbf{E}$, where $\mathrm{id}=\left(I_{n}, I_{n}, \ldots, I_{n}\right) \in \mathbb{C} \subset \mathfrak{g}$. Thus we get an action of $\mathfrak{p g}$ on $\left(D_{U} / D_{U}(1 \otimes \mathbf{E}-n k)\right)^{\mathbb{C}^{*}}=D_{\mathfrak{X}}(n k)$.)

3.6. Recall the Lie algebra $\mathfrak{g}=\operatorname{Lie}(G)$ and its quotient $\mathfrak{p g}=\operatorname{Lie}(P G)$ which is simply $\mathfrak{g} / \mathbb{C} \cdot \mathrm{id}$, where $\mathrm{id}=\left(I_{n}, \ldots, I_{n}\right) \in \mathfrak{g}$. Let $\chi_{c}: \mathfrak{g} \longrightarrow \mathbb{C}$ send an element $(X)=$ $\left(X_{0}, \ldots, X_{\ell-1}\right) \in \mathfrak{g}$ to

$$
\chi_{c}(X)=\sum_{r=0}^{\ell-1} C_{r} \operatorname{Tr}\left(X_{r}\right)
$$

where $C_{r}=\ell^{-1}\left(1-\sum_{m=1}^{\ell-1} \eta^{m r} c_{m}\right)$ for $1 \leq r \leq \ell-1$ and $C_{0}=\ell^{-1}\left(1-\ell-\sum_{m=1}^{\ell-1} c_{m}\right)$. Observe that

$$
\chi_{c}(\mathrm{id})=\operatorname{Tr}\left(I_{n}\right) \sum_{r=0}^{\ell-1} C_{i}=n \sum_{r=0}^{\ell-1} \sum_{m=0}^{\ell-1}-\eta^{r m} c_{m}=0 .
$$

In particular $\chi_{c}$ is actually a character of $\mathfrak{p g}$.

Let $\chi_{k}: \mathfrak{g} \longrightarrow \mathbb{C}$ send an element $(X)=\left(X_{0}, \ldots, X_{\ell-1}\right)$ to $\chi_{k}(c)=k \operatorname{Tr}\left(X_{0}\right)$.

We shall be regularly using the character $\chi_{k, c} \in \mathfrak{g}^{*}$ defined by $\chi_{k, c}=\chi_{c}+\chi_{k}$.

3.7. Let us recall Oblomkov's deformed Harish-Chandra homomorphism [12]. By Lemma 2.4, $\mathcal{S}=\omega\left(\mathfrak{h}^{\text {reg }} / \Gamma_{n}\right)$ is a subset of $\operatorname{Rep}(Q, n \delta)^{r e g}$ which is a slice for the 
$P G$-action on $\operatorname{Rep}(Q, n \delta)$. Let

$$
W_{k}^{\prime}=\left(y_{1} \ldots y_{n}\right)^{-k} \mathbb{C}_{(0)}\left[y_{1}^{ \pm 1}, \ldots, y_{n}^{ \pm 1}\right],
$$

a space of multivalued functions on $\left(\mathbb{C}^{*}\right)^{n}$. The Lie algebra $\mathfrak{g}$ acts on $W_{k}^{\prime}$ by projection onto its 0 -th summand $\mathfrak{g l}(n)$, and then by the natural action of $\mathfrak{g l}(n)$ on polynomials (so that $E_{i j}$ acts as $\left.y_{i} \partial / \partial y_{j}\right)$. With this action the identity matrix in $\mathfrak{g l}(n)$ becomes the Euler operator $\mathbf{E}$ which acts by multiplication by $-n k$. Thus we can make $W_{k}^{\prime}$ a pg-module by twisting $W_{k}^{\prime}$ by the character $\chi_{k}$ since then id acts trivially. If we call this module $W_{k}$, then $W_{k}=W_{k}^{\prime} \otimes \chi_{k}$. Now define $F u n^{\prime}$ to be the space of functions on $\operatorname{Rep}(Q, n \delta)$ of the form

$$
f=\tilde{f} \prod_{i=0}^{\ell-1} \operatorname{det}\left(X_{i}\right)^{r_{i}}
$$

where $\tilde{f}$ is a rational function on $\operatorname{Rep}(Q, n \delta)^{r e g}$ regular on $\mathcal{S}, r_{i}=\sum_{j=0}^{i} C_{j}+\sigma$ and $\sigma=\ell^{-1} \sum_{s=0}^{\ell-1} s C_{s}$. Then $\left(F u n^{\prime} \otimes W_{k}\right)^{\mathfrak{p g}}$ is a space of $\left(\mathfrak{p g}, \chi_{c}\right)$-semi-invariant functions defined on a neighbourhood of $\mathcal{S}$ that take values in $W_{k}$. This space is a free $\mathbb{C}\left[\mathfrak{h}^{\text {reg }}\right]^{\Gamma_{n}}$ module of rank 1 , the isomorphism being given by restriction to $\mathcal{S}$. (Note that the determinant of an element of the form $(X, \ldots, X)$ is $\operatorname{det}(X)^{\sum r_{i}}=1$ as $\sum r_{i}=0$.) Any $\mathfrak{p g}$-invariant differential operator $D$ acts on such a function $f$. Oblomkov defines his homomorphism to be the restriction of $D(f)$ to $\mathcal{S}$.

3.8. We can view the procedure above in terms of $\operatorname{Rep}\left(Q_{\infty}, \epsilon\right)$. By Lemma 2.5 we use $\mathcal{S}_{\infty}=\mathcal{S} \times(1, \ldots, 1) \in U_{\infty}$ as a slice for the $G$-action. The space $\mathcal{S} \times\left(\mathbb{C}^{*}\right)^{n}$ is a closed subset of $U_{\infty}$ since the condition that $i$ be cyclic for $\operatorname{diag}\left(x_{1}, \ldots, x_{n}\right)$ is equivalent to $i \in\left(\mathbb{C}^{*}\right)^{n}$. Thus functions on a neighbourhood of $\mathcal{S}_{\infty}$ in $U_{\infty}$ can be identified with functions from a neighbourhood of $\mathcal{S}$ taking values in functions on $\left(\mathbb{C}^{*}\right)^{n}$. In particular, we can consider elements on $\left(F u n^{\prime} \otimes W_{k}\right)^{\mathfrak{p g}}$ first as $\left(\mathfrak{g}, \chi_{k, c}\right)$-semiinvariant functions from a neighbourhood of $\mathcal{S}$ taking values in $W_{k}^{\prime}$ and hence as $\left(\mathfrak{g}, \chi_{k, c}\right)$-semiinvariant functions on an open set in a neighbourhood of $\mathcal{S}_{\infty}$. We can apply any element of $D \in D\left(U_{\infty}\right)^{\mathfrak{g}}$ to these $\left(\mathfrak{g}, \chi_{k, c}\right)$-semiinvariant functions and then restrict to $S_{\infty}$ to get a homomorphism

$$
\mathfrak{F}_{k, c}: D\left(U_{\infty}\right)^{\mathfrak{g}} \longrightarrow D\left(\mathfrak{h}^{\text {reg }} / \Gamma_{n}\right)
$$

3.9. Since $\operatorname{Rep}\left(Q_{\infty}, \epsilon\right)=\operatorname{Rep}(Q, n \delta) \times \mathbb{C}^{n}$ there is a mapping

$$
\mathfrak{G}: D(\operatorname{Rep}(Q, n \delta))^{\mathfrak{p g}} \longrightarrow D\left(U_{\infty}\right)^{\mathfrak{g}}
$$

that sends $D \in D(\operatorname{Rep}(Q, n \delta))^{\mathfrak{p g}}$ to $(D \otimes 1)$. Oblomkov's homomorphism is $\mathfrak{F}_{k, c} \circ \mathfrak{G}$.

3.10. Differentiating the $G$-action on $U_{\infty}$ gives a Lie algebra homomorphism $\hat{\tau}: \mathfrak{g} \longrightarrow \operatorname{Vect}\left(U_{\infty}\right)$ which we extend to an algebra map

$$
\hat{\tau}: U(\mathfrak{g}) \longrightarrow D\left(U_{\infty}\right) .
$$

By Lemma 2.5, $U_{\infty}$ is a principal $G$-bundle over $\mathfrak{h}^{\text {reg }} / \Gamma_{n}$, and so a generalisation of $\left[14\right.$, Corollary 4.5] shows that the kernel of $\mathfrak{F}_{k, c}$ is $\left(D\left(U_{\infty}\right)\left(\hat{\tau}-\chi_{k, c}\right)(\mathfrak{g})\right)^{\mathfrak{g}}$. Moreover, since the finite group $\Gamma_{n}$ acts freely on $\mathfrak{h}^{\text {reg }}$ we can identify $D\left(\mathfrak{h}^{\text {reg }} / \Gamma_{n}\right)$ with $D\left(\mathfrak{h}^{\text {reg }}\right)^{\Gamma_{n}}$. 
3.11. Recall that

$$
D_{\mathfrak{X}}(n k) \cong\left(\frac{D_{U}}{D_{U}\left(\hat{\tau}-\chi_{k}\right)(\mathbb{C} \cdot \mathrm{id})}\right)^{\mathbb{C}^{*}}
$$

Hence we have

$$
\left(\frac{D_{U}}{D_{U}\left(\hat{\tau}-\chi_{k, c}\right)(\mathfrak{g})}\right)^{G} \cong\left(\frac{D_{\mathfrak{X}}(n k)}{D_{\mathfrak{X}}(n k)\left(\tau-\chi_{c}\right)(\mathfrak{p g})}\right)^{P G},
$$

where $U=\{(X, i): i \neq 0\} \subset \operatorname{Rep}\left(Q_{\infty}, n \delta\right)$ as in 2.7. We consider the restriction mapping $D_{U} \longrightarrow D\left(U_{\infty}\right)$. Composing the global sections of the isomorphism above with this restriction and the homomorphism $\mathfrak{F}_{k, c}$ gives

$$
\mathfrak{R}_{k, c}^{\prime}:\left(\frac{D(\mathfrak{X}, n k)}{D(\mathfrak{X}, n k)\left(\tau-\chi_{c}\right)(\mathfrak{p g})}\right)^{P G} \longrightarrow D\left(\mathfrak{h}^{\text {reg }}\right)^{\Gamma_{n}} .
$$

3.12. Let

$$
\delta_{k, c}(x)=\delta^{-k-1} \delta_{\Gamma}^{\sigma}
$$

where $\delta=\prod_{1 \leq i<j \leq n}\left(x_{i}^{\ell}-x_{j}^{\ell}\right)$ and $\delta_{\Gamma}=\prod_{i=1}^{n} x_{i}$. Define a twisted version of $\mathfrak{R}_{k, c}^{\prime}$ above by

$$
\mathfrak{R}_{k, c}(D)=\delta_{k, c}^{-1} \circ \mathfrak{R}_{k, c}^{\prime}(D) \circ \delta_{k, c}
$$

for any differential operator $D$.

3.13. Our main result is as follows.

THEOREM. For all values of $k$ and $c$, the homomorphism $\mathfrak{R}_{k, c}$ has image $\operatorname{im} \theta_{k, c}$. In particular we have an isomorphism

$$
\theta_{k, c}^{-1} \circ \mathfrak{R}_{k, c}:\left(\frac{D(\mathfrak{X}, n k)}{D(\mathfrak{X}, n k)\left(\tau-\chi_{c}\right)(\mathfrak{p g})}\right)^{\mathfrak{p g}} \stackrel{\sim}{\longrightarrow} U_{k, c} .
$$

Proof. Let us abuse notation by writing $U_{k, c}$ for the image of $U_{k, c}$ in $D\left(\mathfrak{h}^{\text {reg }}\right)^{\Gamma_{n}}$ under $\theta_{k, c}$. Since $\mathfrak{X}=\operatorname{Rep}(Q, n \delta) \times \mathbb{P}^{n-1}$, there is a mapping given by

$$
D(\operatorname{Rep}(Q, n \delta))^{P G} \longrightarrow D(\mathfrak{X}, n k)^{P G} \longrightarrow D\left(\mathfrak{h}^{\text {reg }}\right)^{\Gamma_{n}},
$$

that sends $D \in D(\operatorname{Rep}(Q, n \delta))^{P G}$ to $\mathfrak{R}_{k, c}(D \otimes 1)$. Recall $\tau$ from (3.5.1). Since $\operatorname{gr} \tau=$ $\mu_{\mathcal{X}}^{*}$ we have an inclusion $\operatorname{gr}(D(\mathfrak{X}, n k)) \mu_{\mathcal{X}}^{*}(\mathfrak{p g}) \subseteq \operatorname{gr}\left(D(\mathfrak{X}, n k)\left(\tau-\chi_{c}\right)(\mathfrak{p g})\right)$. This gives a graded surjection

$$
p:\left(\frac{\operatorname{gr} D(\mathfrak{X}, n k)}{\operatorname{gr}(D(\mathfrak{X}, n k)) \mu_{\mathcal{X}}^{*}(\mathfrak{p g})}\right)^{P G} \longrightarrow \operatorname{gr}\left(\frac{D(\mathfrak{X}, n k)}{D(\mathfrak{X}, n k)\left(\tau-\chi_{c}\right)(\mathfrak{p g})}\right)^{P G} .
$$


By Remark 2.7 the composition

$$
\begin{aligned}
\operatorname{gr} D(\operatorname{Rep}(Q, n \delta))^{P G} & \longrightarrow \operatorname{gr} D(\mathfrak{X}, n k)^{P G} \longrightarrow\left(\frac{\operatorname{gr} D(\mathfrak{X}, n k)}{\operatorname{gr}(D(\mathfrak{X}, n k)) \mu_{\mathcal{X}}^{*}(\mathfrak{p g})}\right)^{P G} \\
& \longrightarrow \operatorname{gr}\left(\frac{D(\mathfrak{X}, n k)}{D(\mathfrak{X}, n k)\left(\tau-\chi_{c}\right)(\mathfrak{p g})}\right)^{P G}
\end{aligned}
$$

is surjective. Thus the homomorphism

$$
D(\operatorname{Rep}(Q, n \delta))^{P G} \longrightarrow\left(\frac{D(\mathfrak{X}, n k)}{D(\mathfrak{X}, n k)\left(\tau-\chi_{c}\right)(\mathfrak{p g})}\right)^{P G}
$$

is also surjective. In particular, by 3.9 this implies that the image of $\mathfrak{R}_{k, c}$ equals the image of Oblomkov's Harish-Chandra homomorphism, which, by [12, Theorem 2.5], is $U_{k, c}$.

Thus we have a filtered surjective homomorphism

$$
\mathfrak{R}_{k, c}:\left(\frac{D(\mathfrak{X}, n k)}{D(\mathfrak{X}, n k)\left(\tau-\chi_{c}\right)(\mathfrak{p g})}\right)^{P G} \longrightarrow U_{k, c} .
$$

Thus the dimension of the left hand side is at least $2 \operatorname{dim} \mathfrak{h}=\operatorname{dim} U_{k, c}$. By Proposition 2.7

$$
\left(\frac{\operatorname{gr} D(\mathfrak{X}, n k)}{\operatorname{gr}(D(\mathfrak{X}, n k)) \mu_{\mathcal{X}}^{*}(\mathfrak{p g})}\right)^{P G} \cong \mathbb{C}\left[\mu_{\mathfrak{X}}^{-1}(0)\right]^{P G} \cong \mathbb{C}\left[\mathfrak{h} \oplus \mathfrak{h}^{*}\right]^{\Gamma_{n}}
$$

Hence $p$ is a surjection from a domain of dimension $2 \operatorname{dim} \mathfrak{h}$ onto an algebra of dimension at least $2 \operatorname{dim} \mathfrak{h}$ and so is an isomorphism. It follows that $\left(D(\mathfrak{X}, n k) / D(\mathfrak{X}, n k)\left(\tau-\chi_{c}\right)(\mathfrak{p g})\right)^{\mathfrak{p g}}$ is a domain of dimension $2 \operatorname{dim} \mathfrak{h}$. This implies that $\mathfrak{R}_{k, c}$ is an isomorphism.

\section{Application: Shift functors.}

4.1. The Holland-Schwarz Lemma. We wish to understand the space

$$
\frac{D\left(\operatorname{Rep}\left(Q_{\infty}, \epsilon\right)\right)}{D\left(\operatorname{Rep}\left(Q_{\infty}, \epsilon\right)\right)\left(\hat{\tau}-\chi_{k, c}\right)(\mathfrak{g})} .
$$

As we observed in the proof of Theorem 3.13 there is a natural surjective homomorphism

$$
\frac{\operatorname{gr} D\left(\operatorname{Rep}\left(Q_{\infty}, \epsilon\right)\right)}{\operatorname{gr} D\left(\operatorname{Rep}\left(Q_{\infty}, \epsilon\right)\right) \mu^{*}(\mathfrak{g})} \longrightarrow \operatorname{gr}\left(\frac{D\left(\operatorname{Rep}\left(Q_{\infty}, \epsilon\right)\right)}{D\left(\operatorname{Rep}\left(Q_{\infty}, \epsilon\right)\right)\left(\hat{\tau}-\chi_{k, c}\right)(\mathfrak{g})}\right) .
$$

It turns out that this is an isomorphism.

LEMma (Schwarz, Holland). The homomorphism (4.1.1) is an isomorphism of $\mathbb{C}\left[T^{*} \operatorname{Rep}\left(Q_{\infty}, \epsilon\right)\right]$-modules. flat.

Proof. This is [9, Lemma 2.2] since, by Theorem 2.6 (2), the moment map $\mu$ is 
4.2. This lets us prove the first part of the isomorphism in the statement of Theorem 1.4.

LEMMA. There is an algebra isomorphism

$$
\left(\frac{D\left(\operatorname{Rep}\left(Q_{\infty}, \epsilon\right)\right)}{D\left(\operatorname{Rep}\left(Q_{\infty}, \epsilon\right)\right)\left(\hat{\tau}-\chi_{k, c}\right)(\mathfrak{g})}\right)^{G} \longrightarrow\left(\frac{D(\mathfrak{X}, n k)}{D(\mathfrak{X}, n k)\left(\tau-\chi_{c}\right)(\mathfrak{p g})}\right)^{P G}
$$

Proof. We have a natural pg-equivariant mapping

$$
D\left(\operatorname{Rep}\left(Q_{\infty}, \epsilon\right)\right)^{\mathbb{C}^{*}} \longrightarrow D_{U}^{\mathbb{C}^{*}} \longrightarrow D_{\mathfrak{X}}(n k)
$$

which induces a homomorphism

$$
D\left(\operatorname{Rep}\left(Q_{\infty}, \epsilon\right)\right)^{G} \longrightarrow\left(\frac{D(\mathfrak{X}, n k)}{D(\mathfrak{X}, n k)\left(\tau-\chi_{c}\right)(P G)}\right)^{\mathfrak{p g}}
$$

This is surjective since, as we observed in the proof of Theorem 3.13, the image of $D(\operatorname{Rep}(Q, n \delta))^{P G} \subset D\left(\operatorname{Rep}\left(Q_{\infty}, \epsilon\right)^{G}\right.$ spans the right hand side. By (3.11.1) the kernel of this homomorphism includes the ideal $\left(D(\operatorname{Rep}(Q, \infty), \epsilon)\left(\hat{\tau}-\chi_{k, c}\right)(\mathfrak{g})\right)^{G}$. Hence we have a surjective homomorphism

$$
\left(\frac{D\left(\operatorname{Rep}\left(Q_{\infty}, \epsilon\right)\right.}{D(\operatorname{Rep}(Q, \infty), \epsilon)\left(\hat{\tau}-\chi_{k, c}\right)(\mathfrak{g})}\right)^{G} \longrightarrow\left(\frac{D(\mathfrak{X}, n k)}{D(\mathfrak{X}, n k)\left(\tau-\chi_{c}\right)(\mathfrak{p g})}\right)^{P G}
$$

By Lemma 4.1 and Proposition 2.7, there is an isomorphism

$$
\begin{aligned}
\left(\operatorname{gr} \frac{D\left(\operatorname{Rep}\left(Q_{\infty}, \epsilon\right)\right.}{D(\operatorname{Rep}(Q, \infty), \epsilon)\left(\hat{\tau}-\chi_{k, c}\right)(\mathfrak{g})}\right)^{G} & \cong\left(\frac{\operatorname{gr} D\left(\operatorname{Rep}\left(Q_{\infty}, \epsilon\right)\right)}{\operatorname{gr} D\left(\operatorname{Rep}\left(Q_{\infty}, \epsilon\right)\right) \mu^{*}(\mathfrak{g})}\right)^{G}=\mathbb{C}\left[\mu^{-1}(0)\right]^{G} \\
& =\mathbb{C}\left[\mathfrak{h} \oplus \mathfrak{h}^{*}\right]^{\Gamma_{n}}
\end{aligned}
$$

This shows that the algebra on the left is a domain of dimension $2 \operatorname{dim} \mathfrak{h}$ and so (4.2.1) is also injective, as required.

4.3. Shifting. The previous two lemmas provide us with an interesting series of bimodules. Given a character $\Lambda$ of $G$ we define

$$
B_{k, c}^{\Lambda}=\left(\frac{D\left(\operatorname{Rep}\left(Q_{\infty}, \epsilon\right)\right)}{D\left(\operatorname{Rep}\left(Q_{\infty}, \epsilon\right)\right)\left(\hat{\tau}-\chi_{k, c}\right)(\mathfrak{g})}\right)^{\Lambda}
$$

to be the set of $(G, \Lambda)$-semiinvariants. Thanks to Lemma 4.2 and Theorem 3.13 this is a right $U_{k, c}$-module. Now observe that if $x \in \mathfrak{g}$ and $D \in D\left(\operatorname{Rep}\left(Q_{\infty}, \epsilon\right)\right)^{\Lambda}$ then

$$
[\tau(x), D]=\lambda(x) D
$$


where $\lambda=d \Lambda$. Hence $B_{k, c}^{\Lambda}$ is a left $\left(D\left(\operatorname{Rep}\left(Q_{\infty}, \epsilon\right)\right) / D\left(\operatorname{Rep}\left(Q_{\infty}, \epsilon\right)\right)\left(\hat{\tau}-\chi_{k, c}-\lambda\right)(\mathfrak{g})\right)^{G}$ module and so tensoring sets up a shift functor

$$
\begin{aligned}
& S_{k, c}^{\Lambda}:\left(\frac{D\left(\operatorname{Rep}\left(Q_{\infty}, \epsilon\right)\right)}{D\left(\operatorname{Rep}\left(Q_{\infty}, \epsilon\right)\right)\left(\hat{\tau}-\chi_{k, c}\right)(\mathfrak{g})}\right)^{G}-\bmod \\
& \quad \longrightarrow\left(\frac{D\left(\operatorname{Rep}\left(Q_{\infty}, \epsilon\right)\right)}{D\left(\operatorname{Rep}\left(Q_{\infty}, \epsilon\right)\right)\left(\hat{\tau}-\chi_{k, c}-\lambda\right)(\mathfrak{g})}\right)^{G}-\bmod .
\end{aligned}
$$

4.4. The character group of $G$ is isomorphic to $\mathbb{Z}^{\ell}$ via

$$
\left(i_{0}, \ldots, i_{\ell-1}\right) \mapsto\left(\left(g_{0}, \ldots, g_{\ell-1}\right) \mapsto \prod_{r=0}^{\ell-1} \operatorname{det}\left(g_{r}\right)^{i_{r}}\right) .
$$

Corresponding to the standard basis element $\epsilon_{i}$ is the character $\chi_{i}$ of $\mathfrak{g}$ that sends $X \in \mathfrak{g}$ to $\operatorname{Tr}\left(X_{i}\right)$.

LEMma. The bimodule $B_{k, c}^{\epsilon_{i}}$ above is a $\left(U_{k^{\prime}, c^{\prime}}, U_{k, c}\right)$-bimodule, where $k^{\prime}=k+1$ and $c^{\prime}=c+\left(1-\eta^{-i}, 1-\eta^{-2 i}, \ldots, 1-\eta^{-(\ell-1) i}\right)$.

Proof. Recall that $(k, c)$ corresponds to the character of $\mathfrak{g}$ we called $\chi_{k, c}$ which is defined as

$$
\chi_{k, c}(X)=\left(C_{0}+k\right) \operatorname{Tr}\left(X_{0}\right)+\sum_{j=1}^{\ell-1} C_{j} \operatorname{Tr}\left(X_{j}\right),
$$

where $C_{r}=\ell^{-1}\left(1-\sum_{m=1}^{\ell-1} \eta^{m r} c_{m}\right)$ for $1 \leq r \leq \ell-1$ and $C_{0}=\ell^{-1}\left(1-\ell-\sum_{m=1}^{\ell-1} c_{m}\right)$. We need to calculate $\left(k^{\prime}, c^{\prime}\right)$ so that $\chi_{k, c}+\chi_{i}=\chi_{k^{\prime}, c^{\prime}}$. We have

$$
\begin{aligned}
\left(\chi_{n k, c}+\chi_{i}\right)(X) & =\left(C_{0}+k\right) \operatorname{Tr}\left(X_{0}\right)+\operatorname{Tr}\left(X_{i}\right)+\sum_{j=1}^{\ell-1} C_{j} \operatorname{Tr}\left(X_{j}\right) \\
& =\left(C_{0}^{\prime}+k^{\prime}\right) \operatorname{Tr}\left(X_{0}\right)+\sum_{j=1}^{\ell-1} C_{j}^{\prime} \operatorname{Tr}\left(X_{j}\right)
\end{aligned}
$$

Calculation shows that $k^{\prime}=k+1$ and that if $i=0$ then $C_{j}^{\prime}=C_{j}$ and otherwise

$$
C_{j}^{\prime}=C_{j}+ \begin{cases}-1 & \text { if } j=0 \\ 1 & \text { if } j=i \\ 0 & \text { otherwise }\end{cases}
$$

These unpack to give $c_{m}^{\prime}=c_{m}+1-\eta^{-m i}$.

4.5. Question. Thus for each $0 \leq i \leq \ell-1$ we have a shift functor

$$
S_{i}: U_{k, c}-\bmod \longrightarrow U_{k+1, c^{\prime}}-\bmod
$$

where $c^{\prime}$ is as above. When is this an equivalence of categories? 
REMARK. Shift functors are also constructed in [1] and [15]. Hopefully they agree with the functors here.

\section{REFERENCES}

1. Y. Berest and O. Chalykh, Quasi-invariants of complex reflection groups, in preparation.

2. Y. Berest, P. Etingof and V. Ginzburg, Cherednik algebras and differential operators on quasi-invariants, Duke Math. J. 118, 279-337.

3. W. Crawley-Boevey, Geometry of the moment map for representations of quivers, Compositio Math. 126 (2001), 257-293.

4. W. Crawley-Boevey, Decomposition of Marsden-Weinstein reductions for representations of quivers, Compositio Math. 130 (2002), 225-239.

5. P. Etingof and V. Ginzburg, Symplectic reflection algebras, Calogero-Moser space, and deformed Harish-Chandra homomorphism, Invent. Math. 147 (2002), 243-348.

6. P. Etingof, W. L. Gan, V. Ginzburg and A. Oblomkov, The $\Gamma$-Harish-Chandra homomorphism, RT: 0511489 .

7. W. L. Gan and V. Ginzburg, Almost commuting variety, $\mathcal{D}$-modules, and Cherednik algebras, RT:0409262, I.M.R.N, to appear.

8. I. Gordon and J. T. Stafford, Rational Cherednik algebras and Hilbert schemes I, $A d v$. Math. 198 (2005), 222-274.

I. Gordon and J. T. Stafford, Rational Cherednik algebras and Hilbert schemes II, Duke Math. J., to appear.

9. M. Holland, Quantization of the Marsden-Weinstein reduction for extended Dynkin quivers, Ann. Scient. Ecole Norm. Sup. (1999), 813-834.

10. L. Le Bruyn and C. Procesi, Semisimple representations of quivers, Trans. Amer. Math. Soc. 317 (1990), 585-598.

11. T. Levasseur and J.T. Stafford, The kernel of a homomorphism of Harish-Chandra, Ann. Scient. École. Norm. Sup. 29 (1996), 385-397.

12. A. Oblomkov, Deformed Harish-Chandra homomorphism for the cyclic quiver, RT:0504395, April 2005. 46-64.

13. A. Schofield, General representations of quivers, Proc. London Math. Soc. (3) 65(1992),

14. G. W. Schwarz, Lifting differential operators from orbit spaces, Ann. Scient. École. Norm. Sup. 28 (1995), 253-306.

15. R. Vale, Diagonal coinvariants for $\mathbb{Z}_{m} 2 S_{n}, R T: 0505416$, May 2005. 